\begin{tabular}{|c|l|}
\hline Title & Evaluation of Echinococcus multil ocularis tetraspanins as vaccine candidates against primary alveolar echinococcosis \\
\hline Author(s) & $\begin{array}{l}\text { Dang, Zhisheng; Y agi, Kinpei; Oku, Y uzaburo; Kouguchi, Hirokazu; Kajino, Kiichi; W atanabe, Junichi; Matsumoto, } \\
\text { Jun; Nakao, Ryo; Wakaguri, Hiroyuki; Toyoda, A tsushi; Sugimoto, Chi hiro }\end{array}$ \\
\hline Citation & $\begin{array}{l}\text { Vaccine, 27(52), 7339-7345 } \\
\text { https://doi.org/10.1016/.vaccine.2009.09.045 }\end{array}$ \\
\hline Issue Date & $2009-12$-09 \\
\hline Doc URL & http://hdl.handle.net/2115/42601 \\
\hline Type & article (author version) \\
\hline File Information & VAC27-52_7339-7345.pdf \\
\hline
\end{tabular}

Instructions for use 


\section{Evaluation of Echinococcus multilocularis Tetraspanins as Vaccine Candidates against Primary Alveolar Echinococcosis}

\section{Running title: Echinococcosis Tetraspanin Vaccination}

Zhisheng Dang ${ }^{1,2}$, Kinpei Yagi ${ }^{3}$, Yuzaburo Oku ${ }^{4}$, Hirokazu Kouguchi ${ }^{3}$, Kiichi Kajino ${ }^{1}$, Junichi Watanabe ${ }^{5}$, Jun Matsumoto ${ }^{4,6}$, Ryo Nakao $^{1}$, Hiroyuki Wakaguri ${ }^{7}$, Atsushi Toyoda ${ }^{8}$

${ }^{9}$, Chihiro Sugimoto ${ }^{1} *$

1 Department of Collaboration and Education, Research Center for Zoonosis Control, Hokkaido University, Sapporo 001-0020, Japan

${ }^{2}$ Key Laboratory of Veterinary Parasitology of Gansu Province, State Key Laboratory of Veterinary Etiological Biology, Department of Veterinary Parasitology, Lanzhou Veterinary Research Institute, Chinese Academy of Agricultural Science, Lanzhou 730046, Gansu, PR China

${ }^{3}$ Department of Biological Science, Institute of Public Health, Sapporo 060-0819, Japan

4 Laboratory of Parasitology, Department of Disease Control, Graduate School of Veterinary Medicine, Hokkaido University, Sapporo 060-0818, Japan

${ }^{5}$ Department of Parasitology, Institute of Medical Science, The University of Tokyo, Tokyo 108-8639, Japan 
${ }^{6}$ Laboratory of Medical Zoology, Nihon University College of Bioresource Sciences, Fujisawa 252-8510, Japan

${ }^{7}$ Department of Medical Genome Science, Graduate School of Frontier Science, The University of Tokyo, Tokyo 108-8639, Japan

${ }^{8}$ RIKEN Genomic Sciences Center, Yokohama 230-0045, Kanagawa, Japan

${ }^{9}$ Comparative Genomics Laboratory, National Institute of Genetics Yata 1111, Mishima, Shizuoka 411-8540, Japan

*Corresponding author. Mailing address: Department of Collaboration and Education, Research Center for Zoonosis Control, Hokkaido University, Sapporo 001-0020, Japan. Phone: +81-11-706-5297. Fax: +81-11-706-7370. E-mail: sugimoto@czc.hokudai.ac.jp. 


\section{Abstract}

Echinococcus multilocularis causes an important zoonotic cestode disease. The metacestode stage proliferates in the liver of intermediate hosts including human and rodents and forms multiple cysts. Recently, members of a transmembrane protein tetraspanin (TSP) family have been used as vaccines against schistosomosis, or as diagnostic antigens for cysticercosis. In this study, seven tetraspanins of E. multilocularis, designated as TSP1 to TSP7, were evaluated for their protective potential against primary alveolar echinococcosis. The large extracellular loop (LEL) region of these tetraspanins was cloned from a full-length enriched cDNA library of E. multilocularis metacestodes and expressed in Escherichia coli as a fusion protein with thioredoxin. Recombinant TSPs were applied as vaccines against an E. multilocularis primary experimental infection in BALB/c mice. Cyst lesions in the livers of vaccinated and non-vaccinated mice were counted. The cyst lesion reduction rates induced by the seven tetraspanins in vaccinated vis-à-vis non-vaccinated mice were: $87.9 \%, 65.8 \%, 85.1 \%, 66.9 \%, 73.7 \%, 72.9 \%$ and $37.6 \%$. Vaccination conferred protective rates to mice ranging from $0 \%$ (TSP $5,6,7)$ to maximally $33 \%$ (TSP1, 3). The results indicated that recombinant tetraspanins have varying protective effects against primary alveolar echinococcosis and could be used in vaccine development. 
Key words: Echinococcus multilocularis; Tetraspanin; Vaccine; Oncosphere; Primary

Alveolar Echinococcosis

\section{Introduction}

Echinococcus multilocularis is a cestode parasite, whose larval stage causes hepatic alveolar echinococcosis in humans and rodents, and thus it represents an ecological and public health problem in many countries of the Northern Hemisphere [1]. Although prevention of this tumor-like disease is very important, most studies have concentrated on the parasite's morphology and immunology $[2,3]$. The application of protective proteins as vaccines against other cestode parasites, especially Taenia spp. [4-10] and E. granulosus [11-14] suggests that vaccine development for E. multilocularis is also possible. Great success has been achieved in vaccinating against E. granulosus infection using the recombinant protein EG95 $[12,14]$. In similar studies, it has been reported that recombinant proteins EMY162 [15], EM95 [15, 16] and 14-3-3 [17] protect against primary (egg) E. multilocularis infection.

Recently, many studies have focused on the tetraspanins, a transmembrane protein family, for their multiple functions involved in the coordination of intracellular and intercellular processes, including signal transduction, cell proliferation, adhesion, migration, 
cell fusion, and host-parasite interactions [18]. The host-parasite interactions are thought to be associated with immune evasion [19], which has resulted in the use of tetraspanins as vaccines interfering with the schistosome survival strategy [19-22]. Several tetraspanins, TSP1, TSP2 [19] and Sm23 [20] of Schistosoma mansoni, and SJ23 of S. japonicum [21, 22] have been reported as potential vaccine candidates against schistosomosis. It is notable that these tetraspanins caused varying reductions in different parasitic stages: adult worms (TSP1, TSP2, Sm23 and SJ23), liver eggs/granulomas (TSP1, TSP2 and SJ23) and intestinal/fecal eggs (TSP2 and SJ23).

Although such successes have been achieved using tetraspanins to protect mice against different stages of schistosome infections, no studies have been made on tetraspanins in Echinococcus, especially with a view to exploiting their vaccine potential. As a preliminary study, we evaluated the immunogenicity and protective ability of immunization with seven tetraspanins against $E$. multilocularis infection in a murine model.

\section{Materials and methods}

\subsection{Molecular analysis of TSP amino acid sequences by biological prediction servers}

A full-length enriched cDNA library of E. multilocularis metacestodes (Hokkaido isolate) 
was constructed using a vector-capping method (Hitachi Biotechnologies, Tokyo, Japan) and their 5' ends were sequenced. We identified members of the tetraspanin family by BLAST searching on-line using partial sequences, picked seven representative clones from the library and sequenced them in full. The conserved, crucial parts of these proteins-LEL domain (aa 1 aa 81 in figure 1) were compared with other known tetraspanins from helminthes using the Clustal W2 on-line service. Then we used the MegAlign component of DNAStar programme (Version 4.01 DNASTAR, Madison, Wis.) to construct a phylogenetic tree of transmembrane proteins, including the TSP1 to TSP7 tetraspanins of $E$. multilocularis; the Sm23 [20], Sm25 [23], Sm-TSP1 and Sm-TSP2 proteins of S. mansoni [19]; the $\mathrm{Sj} 23[21,22]$ and $\mathrm{Sj} 25$ proteins of $S$. japonicum [24], the $\mathrm{Sh} 23$ protein of $S$. haematobium [25] and T24 protein of Taenia solium [26]. We also used the DNAStar program to determine the percentage identity of the several TSPs of E. multilocularis.

\subsection{Cloning, expression and purification of recombinant proteins}

For facilitating solubilization and purification of expressed proteins, the regions encoding the extracellular loop (LEL) were amplified by RT-PCR with Taq polymerase to fuse them in-frame with the N-terminal E. coli thioredoxin (TRX) and the C-terminal V5 
and 6-His epitopes in the $\mathrm{pBAD}$ /Thio-TOPO plasmid (Invitrogen, USA). The primers used are listed in Table 1. The cDNAs were ligated into the plasmid and were transformed into $E$. coli TOP10 cells (Invitrogen, USA) with recombinant plasmids according to the manufacturer's instructions (pBAD/TOPO ${ }^{\circledR}$ ThioFusion $^{\mathrm{TM}}$ Expression Kit, Invitrogen, USA). The recombinant bacteria were cultured in Lennox broth at $37^{\circ} \mathrm{C}$ for 16 hours, complemented with $0.05 \%$ arabinose as an inducer. Recombinant fusion proteins from $E$. coli lysates were purified with a HisTrap affinity column under nondenaturing conditions (HisTrap FF crude $1 \mathrm{ml}$, GE Healthcare, USA). The purified proteins were dialyzed in PBS with Snake Skin Pleated Dialysis Tubing (10,000 MWCO, PIERCE, USA) and stored at $-80^{\circ} \mathrm{C}$.

\subsection{Animal experiments}

All animal experiments were performed in accordance with the guidelines of Hokkaido University and Hokkaido Prefecture.

\subsection{Antigen preparation from infected cotton rats}

Cotton rats were infected with the eggs of E. multilocularis (Hokkaido isolate). Nine weeks post-infection, the brood capsules were formed and the protoscolex appeared. Cotton rats were then dissected and cyst blocks were collected from the abdominal cavity, 
removing as much host tissue as possible. After washing several times with $0.15 \mathrm{M}$ PBS (pH7.4), the cyst blocks were cut with scissors and treated to several cycles of freeze/thawing in liquid nitrogen. We then added 9 volumes of PBS (pH7.4), containing protease inhibitors $(5 \mathrm{mM}$ ethylenediaminetetraacetic acid, $5 \mathrm{mM}$ iodoacetamide, $1 \mathrm{mM}$ phenylmethanesulfonyl fluoride, $1 \mu \mathrm{M}$ pepstatin) and 1\% Triton $\mathrm{X}-100$, and homogenized the cyst blocks with a glass-homogenizer and subsequently with a teflon-homogenizer. The tissues were further homogenized using the ultrasonic disruptor (UR-200P, Tomy Seiko, Tokyo, Japan) at $40 \mathrm{~W}$ for $3 \mathrm{~min}$. The lysate was centrifuged at $10,000 \times \mathrm{g}$ for $30 \mathrm{~min}$ at $4^{\circ} \mathrm{C}$ and the supernatant was dialyzed for $48 \mathrm{hrs}$ in PBS (pH 7.4). Dialyzed proteins were finally filtered through a nitrocotton filter $(1 \mu \mathrm{m}$ pore size $)$. The cyst-extracted antigen was stored at $-80^{\circ} \mathrm{C}$.

\subsection{Rabbit immunization and polyclonal antisera generation}

For the studies on specific antibody response and cross-reactivity of the seven tetraspanin proteins, polyclonal anti-sera directed against the seven TSPs were generated in seven 15 weeks old male rabbits. One rabbit for each recombinant TSP was immunized by three successive intramuscular injections of purified recombinant fusion TSP proteins at day 0 , 14 and 28 separately at a dosage of $150 \mu \mathrm{g} /$ rabbit. TSP proteins for primary immunization 
were emulsified in Freund's complete adjuvant, while those for the second and third boosters were emulsified in Freund's incomplete adjuvant. Immunization was completed within six weeks. Pre-immune serum was collected as a negative control and all sera were aliquoted and stored at $-80^{\circ} \mathrm{C}$. For immunoblotting analysis and ELISA tests, the antisera to TSP proteins were absorbed by affinity chromatography with HiTrap affinity columns (HiTrap NHS-activated HP $1 \mathrm{ml}$, GE Healthcare, USA), coupled with purified TRX. This absorption was performed in order to deplete the antisera of antibodies directed to the fusion partner protein TRX.

\subsection{SDS-polyacrylamide gel electrophoresis and immunoblotting}

Recombinant and cyst-extracted proteins were treated with Laemmli sample buffer (BIO-RAD, USA), heated at $98^{\circ} \mathrm{C}$ for $5 \mathrm{~min}$, and then separated on a $12 \%$ SDS-polyacrylamide gel. For immunoblotting analysis, proteins were transferred from the gel to a PVDF membrane (BIO-RAD, USA) using the Trans-Blot ${ }^{\mathrm{TM}}$ SD Semi-Dry Transfer Cell (Bio-Rad, USA). The membrane was blocked with 5\% skimmed milk in TBS-T for 1 hour and was subsequently incubated with the anti-TSP antibody at a dilution of 1:5,000 in $3 \%$ bovine serum albumin/PBS for 1 hour. This was followed by incubation with horseradish peroxidase (HRP)-conjugated goat-anti-rabbit antibody at a dilution of 
1:10,000 in $3 \%$ BSA/PBS for 1 hour. Finally, an HRP substrate Luminol Reagent (Millipore Corporation, USA) was added and the reaction was detected by chemiluminescence using the LAS1000 Mini Reader (FUJIFILM, Japan). Three washes with TBS-T were performed after every step. Immunoblotting was performed at room temperature.

\subsection{Vaccination procedure}

Seventy female BALB/c mice were divided into 10 groups at seven weeks old, each having seven mice. Groups One, Two and Three were non-immunized or immunized with PBS plus adjuvant, and TRX plus adjuvant, respectively as controls, while Groups Four to Ten were immunized with respective TSPs (TSP1 to TSP7) plus adjuvant. For Groups Four to Ten, each mouse received a subcutaneous injection of $20 \mu \mathrm{g}$ of protein dissolved in 100 $\mu 1 \mathrm{PBS}$, and emulsified in an equal volume of Freund's complete adjuvant (primary immunization) and Freund's incomplete adjuvant (booster). PBS and TRX controls were similarly treated by replacing TSP proteins with PBS or TRX. The primary immunization was done on day 0 . Boosters were given twice on days 21 and 42 . Sera were collected and tested for the antibody by ELISA on day 49 post-immunization. One mouse from Group One, Four, Five, Six and Nine died during the etherizing pre-challenge. 


\subsection{Evaluation of vaccine efficacy}

Experimental infection was done using E. multilocularis eggs collected from the feces of an experimentally infected dog. The etherized mice were orally administrated with $0.5 \mathrm{ml}$ of eggs suspension at a concentration of 400 eggs per $\mathrm{ml}$ in physiological saline 56 days post-immunization. One month post-infection, all mice were sacrificed and necropsies were performed. Livers were collected in plastic dishes and cut into approximately $0.5 \mathrm{~mm}$ thick slices, from which the numbers of all detectable lesions were counted. Based on these data, the cyst reduction rate of each tetraspanin was calculated as a percentage reduction in cyst lesion counts in vaccinated groups as compared to non-vaccinated ones.

\subsection{Cross-reaction among TSPs determined by ELISA}

We used ELISA to analyze sera from immunized rabbits for their cross reactivity to heterologous TSPs. Prior to ELISA, the sera were absorbed with TRX as described above. Ninety-six well Costar ${ }^{\circledR}$ plates (Corning Inc., USA) were coated with recombinant TSPs at a concentration of $0.1 \mu \mathrm{g} / \mathrm{ml}$ in carbonate buffer ( $\mathrm{pH}$ 9.6) for $2 \mathrm{hrs}$ at room temperature. This was followed by blocking with $5 \%$ skimmed milk overnight at $4{ }^{\circ} \mathrm{C}$ and washing four times with PBS-Tween. Then serum samples, diluted 1: 5,000 in 3\% BSA/PBS, were added 
and the plates were incubated for 1 hour at $37^{\circ} \mathrm{C}$ before washing four times. Goat-anti-rabbit IgG conjugated with horseradish peroxidase (Invitrogen, USA), diluted $1: 10,000$ in $3 \%$ BSA/PBS, was then added and incubated at $37^{\circ} \mathrm{C}$ for 1 hour. This was followed by washing four times and the addition of 3, 3', 5, 5'-tetramethylbenzidine substrate before incubation for $20 \mathrm{~min}$ at room temperature. The reaction was stopped using 1N HCL and color development was measured at $450 \mathrm{~nm}$ with the Biotrack II plate reader (Amersham Biosciences, USA). Antisera to TSP proteins were absorbed with TRX-coupled HiTrap affinity columns (HiTrap NHS-activated HP $1 \mathrm{ml}$, GE Healthcare, USA) deplete the antibodies directed to the fusion partner protein TRX. Pre-immune serum was used as a negative control. The mean OD value of pre-immune sera was calculated and used as a cut-off point (threshold value) to discriminate between "positive" and "negative".

\subsection{Detection of TSPs transcripts in oncosphere by RT-PCR}

Eggs were treated with artificial gastric fluid for 1 hour at $37^{\circ} \mathrm{C}$ and centrifuged at $1,000 \times \mathrm{g}$ for $5 \mathrm{~min}$. The pellet was used to extract total RNA using a TRIzol plus RNA Purification Kit (Invitrogen, USA), and cDNA synthesis was carried out using the SuperScript III First-strand Synthesis System Kit (Invitrogen, USA) according to the manufacturer's instructions. Sense and anti-sense primers (Table 1) were designed to 
amplify tetraspanin open reading frames (ORFs) and RT-PCR was performed with GoTaq Flexi DNA Polymerase (Promega, USA).

\subsection{Data analysis}

The protective effect obtained by immunization with each tetraspanin was calculated as the percentage reduction of the number of cyst lesions in vaccinated mice as compared to non-vaccinated ones. Multiple comparison analysis was performed with the Tukey-Kramer method using a computer package (Statcel 2, Japan) to determine differences between the vaccinated groups and control groups, which were considered significant at the $5 \%$ level.

\section{Results}

\subsection{Molecular analysis of $E$. multilocularis tetraspanin}

Molecular components analysis of LEL domain using the on-line Clustal W2 program revealed that TSP1 to TSP7, of E. multilocularis, share many conserved residues with each other, and with those of Schistosoma and Taenia (Fig. 1), which has been reported previously [27]. The amino acid sequence of these residues was as follows: E/Q $\cdots C C G \cdots D \cdots W / F / Y \cdots P \cdots S C C \cdots C \cdots G C$. The percentage conservation of the amino acids CCG, the first $\mathrm{C}$ of SCC and the terminal C, of TSP1 to 7, was 100\%. Very interestingly, we found that residues $\mathrm{F} / \mathrm{L}$ at aa $5, \mathrm{G}$ at aa 12 and $\mathrm{P} / \mathrm{Y}$ at aa 13 are extremely conserved in 
these helminths, while some residues only exist in Taenia and Echinococcus: A at aa 55, G at aa 69 and aa 81. Percentage identity analysis of the seven TSPs showed that the amino acid sequence identity ranged from $12.6 \%$ to $57.7 \%$ (Table 2 ). In addition, the phylogenetic relationship revealed that TSP3 and TSP5 are closely related to T24, a diagnostic antigen of T. solium; and TSP2 is related to the protective proteins $\mathrm{Sm} 23, \mathrm{Sh} 23, \mathrm{Sj} 23$ and TSP2 of Schistosoma (Fig. 2).

\subsection{Amplified fragments of tetraspanins}

Using the primers listed in Table 1, the LELs of the seven tetraspanins from cyst cDNA and the entire ORFs of the seven tetraspanins from oncosphere cDNA were amplified by PCR. The results showed that the amplified fragment sizes of the LELs ranged from $195 \mathrm{bp}$ to $330 \mathrm{bp}$ (Fig. 3A) and those of the tetraspanin ORFs ranged from $447 \mathrm{bp}$ to $771 \mathrm{bp}$ as expected (Fig. 3B). Only a very weak band of oncosphere TSP1 ORF could be detected (Fig. 3B).

\subsection{SDS-PAGE analysis of recombinant tetraspanins}

SDS-polyacrylamide gel electrophoresis (SDS-PAGE) of the purified fusion proteins showed the expected molecular masses, which ranged from 24 to $26 \mathrm{kDa}$ (Fig. 4). Purified recombinant proteins were soluble and pure enough for further immunological analysis and 
vaccine trials in mice.

\subsection{Anti-TSPs IgG antibody response}

Sera from immunized rabbits were examined for the presence of anti-TSP antibodies against TSPs using immunoblotting. Western blot analysis showed that anti-TSP antibodies had very strong responses to cyst-extracted antigens (Fig. 5). Double bands can be found to TSP3. In contrast, pre-immune serum was not reactive with either recombinant or cyst-extracted proteins. The immunostained bands were approximately 23 to $29 \mathrm{kDa}$. Cross-reaction investigation using ELISA showed that TSP1 and TSP3 could induce highly specific antibody responses, while the antibody to TSP2 was strongly cross-reactive with other TSPs including TSP4 to 7. Antisera to TSP4, TSP5, TSP6 and TSP7 showed cross-reactivity with the others to varying extents. According to mean OD value to pre-immune sera (threshold value), all the OD value to anti-TSPs were determined as "positive" (Table 3).

ELISA results using individual sera collected from vaccinated mice showed that although there were many variations among mice within each group and between groups, relatively strong antibody responses were observed against recombinant tetraspanins and cyst-extracted proteins (Fig. 6). 


\subsection{Protective efficacy of tetraspanin proteins}

The number of counted cyst lesions in the liver and the reduction rate of cyst lesions for the seven TSPs (TSP1 to TSP7) are shown in Table 4. The recombinant proteins TSP1, TSP2, TSP3, TSP4, TSP5 and TSP6 gave statistically significant reductions of more than $65 \%(\mathrm{P}<0.05)$ in the number of cyst lesions in vaccinated mice as compared to non-vaccinated ones. However, TSP7 protein did not produce a significant reduction in cyst lesions (37.6\%) ( $\mathrm{P}>0.05)$. PBS showed no vaccine efficacy, while TRX displayed a 27.8\% reduction in cyst lesions $(\mathrm{P}>0.05)$. Among the vaccinated groups, two out of six in the TSP1-vaccinated group, one out of six in the TSP2-vaccinated group, two out six in TSP3-vaccinated group, and one out of seven TSP4-vaccinated group showed no cyst lesions in the liver (Table 4). In consequence, vaccination conferred an overall protection rate against infection ranging from $0 \%$ (TSP5, 6,7$)$ to maximally $33 \%$ (TSP1, 3 ).

\section{Discussion}

Despite high expression levels in the plasma membrane, and intracellular vesicles, tetraspanins remain among the most mysterious transmembrane molecules 20 years after their discovery [28]. To date, several tetraspanins have been identified in helminths, 20 in Caenorhabditis elegans and at least 25 in the parasite Schistosoma [29]. Although some 
tetraspanins have been demonstrated to be potential vaccine candidates against different stages of schistosome infection [19-22], nothing is known about them in another important platyhelminth E. multilocularis. Recently, many studies have focused on the LEL domain of tetraspanin because of its important functions in mediating protein-protein interactions and homodimerization [30]. In our present study, LEL domain of seven tetraspanins were cloned, expressed and characterized in order to focus on the protective potentials of tetraspanins against Echinococcus infection.

Tetraspanins belong to a four transmembrane protein family, nearly all members of which share 26 conserved key residues. These conserved residues have been used as a key criterion to define whether a newly identified membrane protein is a member of tetraspanin family or not $[27,31]$. Thirteen out of 26 conserved residues are within LEL domain. Molecular component analysis of LEL domain, using the Clustal W2 on-line biological service, indicated that these key residues were conserved in TSP proteins of $E$. multilocularis, S. mansoni, S. japonicum and T. solium. Most importantly, we found that among those proteins the crucial cysteine-cysteine-glycine (CCG) motif has no mutations as previously reported [27]. Three extra conserved sites, F/L at aa 5, G at aa 12 and $\mathrm{P} / \mathrm{Y}$ at aa 13, were found in tetraspanins of these helminths (Figure 1), implying the possibly 
closer relationship of these helminths than others. Moreover, residues $A$ at aa 55, G at aa 69 and aa 81 appear to be more conserved in cestode Echinococcus and Taenia. The amino acid sequence analysis of the seven TSPs displayed a relatively high percentage identity ranging from $12.6 \%$ to $57.7 \%$. This is mainly due to the existence of the largest, conserved domain, known as the large extracellular loop (LEL). Members of the tetraspanin family from different organisms are structurally and functionally related [18, 31]. Phylogenetic analysis indicated that TSP2 of E. multilocularis is phylogenetically related to TSP2 [15], Sm23 [20] and SJC23 [21, 22] of Schistosoma, the protective antigens against schistosomiasis, while TSP5 is closely related to the T24 antigen of T. solium, a diagnostic antigen for cysticercosis [26]. We suppose that among these proteins, some may be vaccine candidates, while others may be useful as diagnostic antigens.

Cyst-extracted antigens had strong reactivity with TRX-absorbed rabbit anti-TSP antibodies as determined by immunoblot assay (Fig. 5 and Fig 6) similar to that of recombinant proteins (Fig. 6), suggesting a well antigenicity of TSP proteins. The much closed double bands detected to TSP3 indicated possible differential glycosylation (Fig. 5).

Since tetraspanins in the same family share common crucial residues [27, 31], antigenic cross-reactivity would be expected between the different TSP molecules. The ELISA 
results indicated that recombinant tetraspanin proteins showed varying degrees of cross-reactivity with each other. Although all the OD values to anti-TSPs sera were determined as "positive" referencing to threshold value, anti-TSP1 and anti-TSP3 antibodies exhibited the highest specific reactivities to homologous antigens but had low cross-reactivities with others (Table 3). On the other hand, TSP2 and TSP7 showed a broad range of specificity, while TSP4, TSP5 and TSP6 cross-reacted with other members of the family to varying extents.

Tetraspanin proteins regulate cell morphology, motility, invasion, fusion and signaling, in the brain, immune system, in tumors and elsewhere [32-37], and the most distinctive feature of the tetraspanin family is the ability of its members to form lateral associations with multiple partner proteins, and with each other, in a dynamic assembly, described as the 'tetraspanin web' $[34,38]$. The fact that some tetraspanin proteins cross-reacted with several others implies that immunization with one tetraspanin antigen could block several tetraspanins functions or disrupt the lateral associations with multiple partner proteins and with each other in the tetraspanin web, thereby providing a more effective protection mechanism.

Primary infection, initiated through the oral E. multilocularis egg infection of mice, 
followed by counting liver cyst lesions four weeks post-infection, revealed that six out of seven TSPs had a cyst lesion reduction rate of over $65 \%$ in this study. TSP 1 and TSP3 exhibited reduction rates of $87.9 \%$ and $85.1 \%$, respectively, while TSP7 had the poorest protective effect of $37.4 \%$. The most encouraging results were found in the groups for TSP1 to 4, in which the number of liver cyst lesions in some mice was found to be zero, indicating that these mice were completely protected against E. multilocularis. Compared to the non-vaccinated group, the PBS plus adjuvant group showed no protective efficacy, while TRX plus adjuvant had a $27.8 \%$ reduction in cyst lesions.

Much success has been achieved in the vaccination campaign against E. granulosus infection using the recombinant protein EG95 $[12,14]$. In similar studies, Kouguchi et al. [15] reported $74.3 \%$ protection against E. multilocularis infection in mice using the recombinant protein EMY162, and Gauci et al. [16] and Kouguchi et al. [15] reported 79\% and $82.9 \%$ protection respectively, against E. multilocularis infection in mice using the recombinant protein EM95. In addition, the recombinant protein 14-3-3 was reported to have $97 \%$ protection against a primary infection of E. multilocularis [17]. It can be seen from the above results that the reduction in liver cysts for EMY162 and EM95 were lower than for TSP1 $(87.9 \%)$ and TSP3 (85.1\%). Moreover, secondary (intraperitoneal) infection 
has not been performed to evaluate the vaccination efficacy with these proteins. Although the recombinant protein 14-3-3, showed a higher protection against primary infection than those used in this study, showed no protection against secondary infections in mice. As reported, except for erythrocytes, all cells seem to express several tetraspanins [39-41] and individual tetraspanin proteins are often expressed in large copy numbers per cell [33]. Additionally, as mentioned above, tetraspanins have showed their potential in protecting a host against different stages of Schistosoma infection [19-22]. We believe that the discovery of one, or more tetraspanins, which are expected to have 'broad-spectrum protection' against different stages of E. multilocularis infections, will be achieved in our future studies.

Our RT-PCR results showed that transcripts for these TSPs exist in both the metacestode and oncosphere stages of E. multilocularis. Moreover, our study on TSP localization revealed that most of these TSPs are expressed on the germinal layer of E. multilocularis metacestodes (in press). Usually, the tegument of E. multilocularis metacestodes is thought to be the outer part of the germinal layer [42, 43]; therefore, we speculate that TSPs on the Echinococcus germinal layer/surface play crucial roles in parasite survival and parasite-host interactions. Proteins expressed on the surface, as membrane-integrated proteins, have a 
wide range of functions in parasite biology [44]. Loukas et al. [45] emphasized the importance of the schistosome tegument/body wall by describing it as 'the key to its success but also its Achilles heel', because the schistosome tegument has been described as being responsible for parasite survival through immune evasion, while from a vaccine perspective, it constitutes the host-parasite interface. Although we are unsure, in our experiments, whether the antibodies raised by these tetraspanins kill the metacestode or the oncosphere, or even a parasite stage in between, previous studies with schistosomes suggested that once the surface of the parasite is blocked by anti-TSP antibodies, they will lose their survival strategies and render the parasite surface vulnerable to the host defense mechanisms [19].

In this study, we are the first to identify and characterized a new tetraspanin family as vaccine candidates with high protection potential against primary E. multilocularis infection in mice. The numerous properties displayed by tetraspanins, and the results from our studies, call for in-depth analyses to explore their vaccine potential in protecting intermediate and final hosts against different stages of infections.

\section{Acknowledgements}

Many thanks are addressed to Mr. T. Ichihashi for advice on animal care and 
immunization. Thanks to Dr. J. W. Magona and Miss S. L. Wastling (University of Edinburgh) for helpful discussions and critical reading of the manuscript. This work was supported by Grant-in-Aid for Scientific Research from the Ministry of Education, Culture, Sports, Science and Technology of Japan (MEXT) and the program of Funding Research Center for Emerging and Re-emerging Infectious Disease, MEXT.

\section{References}

1. Matsumoto J, Yagi K. Experimental studies on Echinococcus multilocularis in Japan, focusing on biohazardous stages of the parasite. Exp Parasitol 2008; 119(4): 534-41.

2. Hemphill A, Gottstein B. Immunology and morphology studies on the proliferation of in vitro cultivated Echinococcus multilocularis metacestodes. Parasitol Res 1995; 81(7): $605-14$

3. Sakamoto T, Sugimura M. Studies on echinococcosis. 23. Electron microscopical observations on histogenesis of larval Echinococcus multilocularis. Jpn J Vet Res 1970; 18(3): 131-44.

4. Cai X, Yuan G, Zheng Y, Luo X, Zhang S, Ding J, et al. Effective production and purification of the glycosylated TSOL18 antigen, which is protective against pig 
cysticercosis. Infect Immun 2008; 76(2): 767-70.

5. Guo A, Jin Z, Zheng Y, Hai G, Yuan G, Li H, et al. Induction of protection against porcine cysticercosis in growing pigs by DNA vaccination. Vaccine 2007; 25(1): 170-5.

6. Sciutto E, Rosas G, Hernández M, Morales J, Cruz-Revilla C, Toledo A, et al. Improvement of the synthetic tri-peptide vaccine (S3Pvac) against porcine Taenia solium cysticercosis in search of a more effective, inexpensive and manageable vaccine. Vaccine 2007; 25(8): 1368-78.

7. Gauci C, Vural G, Öncel T, Varcasia A, Damian V, Kyngdon CT. et al. Vaccination with recombinant oncosphere antigens reduces the susceptibility of sheep to infection with Taenia multiceps. Int J Parasitol 2008; 38(8-9): 1041-50.

8. Johnson KS, Harrison GBL, Lightowlers MW, O’Hoy KL, Cougle WG, Dempster RP, et al. Vaccination against ovine cysticercosis using a defined recombinant antigen. Nature 1989; 338(6216): 585-7.

9. Lightowlers MW, Rolfe R, Gauci CG. Taenia saginata: vaccination against cysticercosis in cattle with recombinant oncosphere antigens. Exp Parasitol 1996; 84(3): 330-8.

10. Plancarte A, Flisser A, Gauci CG, Lightowlers MW. Vaccination against Taenia solium cysticercosis in pigs using native and recombinant oncosphere antigens. Int $\mathrm{J}$ Parasitol. 
1999; 29(4): 643-7.

11. Heath DD, Lawrence SB. Antigenic polypeptides of Echinococcus granulosus oncospheres and definition of protective molecules. Parasite Immunol 1996; 18(7): 347-57.

12. Lightowlers MW, Jensen O, Fernandez E, Iriarte JA, Woollard DJ, Gauci CG, et al. Vaccination trials in Australia and Argentina confirm the effectiveness of the EG95 hydatid vaccine in sheep. Int J Parasitol 1999; 29(4): 531-4.

13. Petavy AF, Hormaeche C, Lahmar S, Ouhelli H, Chabalgoity A, Marchal T, et al. An oral recombinant vaccine in dogs against Echinococcus granulosus, the causative agent of human hydatid disease: A pilot study. PLoS Negl Trop Dis 2008; 2(1): e125.

14. Woollard DJ, Gauci CG, Heath DD, Lightowlers MW. Protection against hydatid disease induced with the EG95 vaccine is associated with conformational epitopes. Vaccine 2000; 19(4-5): 498-507.

15. Kouguchi H, Matsumoto J, Katoh Y, Okun Y, Suzuki T, Yagi K. The vaccination potential of EMY162 antigen against Echinococcus multilocularis infection. Biochem. Biophys Res Commun 2007; 363(4): 915-20.

16. Gauci C, Merli M, Muller V, Chow C, Yagi K, Mackenstedt U, et al. Molecular cloning of a vaccine antigen against infection with the larval stage of Echinococcus multilocularis. Infect Immun 2002; 70(7): 3969-72.

17. Siles-Lucas M, Merli M, Mackenstedt U, Gottstein B. The Echinococcus multilocularis 
14-3-3 protein protects mice against primary but not secondary alveolar echinococcosis. Vaccine 2003; 21(5-6): 431-9.

18. Levy S, Shoham T. Protein-protein interactions in the tetraspanin web. Physiology (Bethesda) 2005; 20(4): 218-24.

19. Tran MH, Pearson MS, Bethony JM, Smyth DJ, Jones MK, Duke M, et al. Tetraspanins on the surface of Schistosoma mansoni are protective antigens against schistosomiasis. Nat Med 2006; 12(7): 835-40.

20. Da'dara AA, Skelly PJ, Wang MM, Harn DA. Immunization with plasmid DNA encoding the integral membrane protein, Sm23, elicits a protective immune response against schistosome infection in mice. Vaccine 2001; 20(3-4): 359-69.

21. Da'dara AA, Li Y, Xiong T, Zhou J, Williams GM, McManus DP, et al. DNA-based vaccines protect against zoonotic schistosomiasis in water buffalo. Vaccine 2008; 26(29-30): $3617-25$

22. Zhu Y, Ren J, Da'dara A, Harn D, Xu M, Si J, et al. The protective effect of a Schistosoma japonicum Chinese strain $23 \mathrm{kDa}$ plasmid DNA vaccine in pigs is enhanced with IL-12. Vaccine 2004; 23(1): 78-83.

23. Pearce EJ, Magee AI, Smithers SR, Simpson AJ. Sm25, a major schistosome tegumental glycoprotein, is dependent on palmitic acid for membrane attachment. EMBO J 1991; 10(10): 2741-6. 
24. Fan J, Brindley PJ. Characterization of cDNAs encoding a new family of tetraspanins from schistosomes-the Sj25 family. Gene1998; 219(1-2): 1-8.

25. Inal J, Bickle Q. Sequence and immunogenicity of the 23-kDa transmembrane antigen of Schistosoma haematobium. Mol Biochem Parasitol 1995; 74(2): 217-21.

26. Hancock K, Pattabhi S, Whitfield FW, Yushak ML, Lane WS, Garcia HH, et al. Characterization and cloning of T24, a Taenia solium antigen diagnostic for cysticercosis. Mol Biochem Parasitol 2006; 147(1): 109-17.

27. Hemler ME. Specific tetraspanin functions. J Cell Biol 2001; 155(7): 1103-7.

28. Charrin S, Le Naour F, Silvie O, Milhiet PE, Boucheix C, Rubinstein E. Lateral organization of membrane proteins: tetraspanins spin their web. Biochem J 2009; 420(2): $133-54$

29. Huang S, Yuan S, Dong M, Su J, Yu C, Shen Y, et al. The phylogenetic analysis of tetraspanins projects the evolution of cell-cell interactions from unicellular to multicellular organisms. Genomics 2005; 86(6): 674-84.

30. Hemler ME. Tetraspanin functions and associated microdomains. Nat Rev Mol Cell Biol 2005; 6(10):801-11.

31. Stipp CS, Kolesnikova TV, Hemler ME. Functional domains in tetraspanin proteins. Trends Biochem Sci 2003; 28(2): 106-12. 
32. Tarrant JM, Robb L, van Spriel AB, Wright MD. Tetraspanins: molecular organisers of the leukocyte surface. Trends Immunol 2003; 24(11): 610-7.

33. Hemler ME. Tetraspanin proteins mediate cellular penetration, invasion and fusion events, and define a novel type of membrane microdomain. Annu Rev Cell Dev Biol 2003; 19: 397-422.

34. Boucheix C, Rubinstein E. Tetraspanins. Cell Mol Life Sci 2001; 58(9): 1189-205.

35. Boucheix C, Thien Duc GH, Jasmin C, Rubinstein E. Tetraspanins and malignancy. Expert Rev Mol Med 2001; 3(4): 1-17.

36. Levy S, Todd SC, Maecker HT. CD81 (TAPA-1): a molecule involved in signal transduction and cell adhesion in the immune system. Annu Rev Immunol 1998; 16: 89-109.

37. Maecker HT, Todd SC, Levy S. The tetraspanin superfamily: molecular facilitators. FASEB J 1997; 11(6): 428-42.

38. Rubinstein E, Le Naour F, Lagaudriere-Gesbert C, Billard M, Conjeaud H, Boucheix C. CD9, CD63, CD81, and CD82 are components of a surface tetraspan network connected to HLA-DR and VLA integrins. Eur J Immunol 1996; 26(11): 2657-65.

39. Sincock PM, Fitter S, Parton RG, Berndt MC, Gamble JR, Ashman LK. PETA-3/CD151, a member of the transmembrane 4 superfamily, is localised to the plasma membrane and endocytic system of endothelial cells, associates with multiple integrins and 
modulates cell function. J Cell Sci 1999; 112(Pt6): 833-44.

40. Boucheix C, Perrot JY, Mirshahi M, Giannoni F, Billard M, Bernadou A, et al. A new set of monoclonal antibodies against acute lymphoblastic leukemia. Leuk Res 1985; 9(5): 597-604.

41. Sincock PM, Mayrhofer G, Ashman LK. Localization of the transmembrane 4 superfamily (TM4SF) member PETA-3 (CD151) in normal human tissues: comparison with CD9, CD63 and alpha5beta1 integrin. J Histochem Cytochem 1997; 45(4): 515-25.

42. Gottstein B, Hemphill A. Echinococcus multilocularis: The parasite-host interplay. Exp Parasitol 2008; 119(4): 447-52.

43. Dai WJ, Waldvogel A, Siles-Lucas M, Gottstein B. Echinococcus multilocularis proliferation in mice and respective parasite 14-3-3 gene expression is mainly controlled by an $\alpha \beta^{+} \mathrm{CD}^{+} \mathrm{T}$-cell-mediated immune response. Immunology 2004; 112(3): 481-8.

44. Rosenzvit MC, Zhang W, Motazedian H, Smyth D, Pearson M. Identification of membrane-bound and secreted proteins from Echinococcus granulosus by signal sequence trap. Int J Parasitol 2006; 36(1): 123-30.

45. Loukas A, Tran M, Pearson MS. Schistosome membrane proteins as vaccines. Int J Parasitol 2007; 37(3-4): 257-63. 
Table 1. Oligonucleotide primers for amplification of the LEL domain and TSP ORFs.

\begin{tabular}{|c|c|c|c|c|}
\hline \multirow[t]{2}{*}{ Primer $^{\mathrm{a}}$} & \multicolumn{4}{|c|}{ Sequence $^{\mathrm{b}}$} \\
\hline & LEL domain & Product (bp) & TSP ORF & Product (bp) \\
\hline TSP1-s & CCTGATAACCTAAACAAAGC & 330 & AGCTATCTTCTCGGATGTGCTAGAC & 792 \\
\hline TSP1-a & GAGGGTTTTGTTCTCTGCCAA & & TTCCGCTTTCCACTGCTTGTACAGC & \\
\hline TSP2-s & TACAAGGATGAGTTGATGGAC & 195 & GGTGTTCTTCAAGGTGGAATGAAGTG & 621 \\
\hline TSP2-a & CATGGAGGACTCAACTTTGCC & & CGCATAACTTCG AATTTTGGACTGC & \\
\hline TSP3-s & CATGAGTTTGTCGGTCTTGTG & 264 & CTGAAGACGTTCGCGGTCATTGT & 447 \\
\hline TSP3-a & CAGAGAGGAATCCTTAATTTG & & CTTCTCGTATTCGTTGACCTTCTTGGCC & \\
\hline TSP4-s & TTCGCGCTGAAAAATCAGTCC & 267 & GCAATGCTGCGCTTTAGCGGTTGCT & 687 \\
\hline TSP4-a & AGGTAGTCTATTTTCCGTCAA & & GGTTACAGATGCTGTCCTAGGGCCAA & \\
\hline TSP5-a & CACGACTTCGTTCGTCTTGTT & 261 & GCTCTCTCTTGCGGTGGTAACTT & 678 \\
\hline TSP5-s & CAGGGCAGAATCCTTTATCTC & & GACCTTCTCATATTCGCCGACCTTCTTG & \\
\hline TSP6-s & GACAAGCAGGGTAAAGACTTT & 252 & GTTCTGACATGCGGAGAGCAGT & 669 \\
\hline TSP6-a & GCTTCGAAGGTATTCGTAGAT & & GCTCCGCTTGCCGGCCAAACAGCA & \\
\hline TSP7-s & GTTTTCAAAGATGAGGTGCGA & 249 & TCGAAAACGTGTGCTGGTTGTATCAG & 690 \\
\hline TSP7-a & CAACGTATGTTTCTCAАTCTC & & CACAGGTTCATAAGATTCAATGTCGG & \\
\hline
\end{tabular}

${ }^{\mathrm{a}} \mathrm{s}$, sense; a, antisense.

${ }^{\mathrm{b}}$ Sequences are 5' to 3 '. 
Table 2. Percentage identity analysis of the entire ORF of TSP by DNASTAR (Version 4.0)

\begin{tabular}{lllllllll}
\hline 1 & 2 & 3 & 4 & 5 & 6 & 7 & \\
\hline 100 & 13.0 & 17.4 & 16.2 & 15.9 & 12.6 & 15.2 & 1 & TSP1 \\
& 100 & 23.5 & 18.4 & 29.0 & 23.7 & 18.4 & 2 & TSP2 \\
& \multirow{2}{*}{100} & 24.2 & 57.7 & 26.8 & 16.8 & 3 & TSP3 \\
& & 100 & 30.1 & 30.5 & 16.6 & 4 & TSP4 \\
& & & 100 & 30.0 & 21.7 & 5 & TSP5 \\
& & & & 100 & 19.3 & 6 & TSP6 \\
& & & & & 100 & 7 & TSP7 \\
\hline
\end{tabular}

Amino acid sequences were used for the percentage identity analysis of TSP proeins. 
Table 3. Cross-reaction of anti-TSP-sera with TSPs by ELISA

\begin{tabular}{l|lllllll}
\hline \multicolumn{1}{r|}{ Protein $^{\mathrm{a}}$} & TSP1 & TSP2 & TSP3 & TSP4 & TSP5 & TSP6 & TSP7 \\
\cline { 1 - 6 } Antibody $^{\mathrm{b}}$ & & & & & & & \\
\hline Anti-TSP1 & $\mathbf{1 . 9 0 3}$ & $\mathbf{0 . 5 7 3}$ & $\mathbf{0 . 2 3 7}$ & $\mathbf{0 . 1 7 2}$ & $\mathbf{0 . 2 2 4}$ & $\mathbf{0 . 1 5 4}$ & $\mathbf{0 . 1 5 4}$ \\
Anti-TSP2 & $\mathbf{1 . 8 7 8}$ & $\mathbf{2 . 3 4 5}$ & $\mathbf{2 . 1 1 5}$ & $\mathbf{0 . 3 1 1}$ & $\mathbf{2 . 0 1 1}$ & $\mathbf{1 . 7 3 0}$ & $\mathbf{1 . 6 4 9}$ \\
Anti-TSP3 & $\mathbf{0 . 1 8 2}$ & $\mathbf{0 . 2 9 3}$ & $\mathbf{2 . 3 0 1}$ & $\mathbf{0 . 1 4 7}$ & $\mathbf{0 . 7 3 1}$ & $\mathbf{0 . 1 4 9}$ & $\mathbf{0 . 1 5 5}$ \\
Anti-TSP4 & $\mathbf{0 . 6 5 6}$ & $\mathbf{2 . 7 1 3}$ & $\mathbf{2 . 2 5 7}$ & $\mathbf{2 . 5 5 9}$ & $\mathbf{0 . 7 2 0}$ & $\mathbf{1 . 3 9 3}$ & $\mathbf{0 . 8 8 7}$ \\
Anti-TSP5 & $\mathbf{0 . 5 6 8}$ & $\mathbf{1 . 0 2 3}$ & $\mathbf{0 . 5 9 4}$ & $\mathbf{0 . 5 3 1}$ & $\mathbf{1 . 3 6 7}$ & $\mathbf{0 . 4 3 2}$ & $\mathbf{0 . 4 4 0}$ \\
Anti-TSP6 & $\mathbf{0 . 5 2 4}$ & $\mathbf{1 . 7 1 7}$ & $\mathbf{0 . 8 4 8}$ & $\mathbf{0 . 2 6 8}$ & $\mathbf{0 . 5 4 3}$ & $\mathbf{1 . 9 0 8}$ & $\mathbf{0 . 1 8 1}$ \\
Anti-TSP7 & $\mathbf{0 . 4 6 3}$ & $\mathbf{1 . 2 6 5}$ & $\mathbf{0 . 8 0 1}$ & $\mathbf{0 . 3 4 4}$ & $\mathbf{0 . 3 7 5}$ & $\mathbf{0 . 3 5 9}$ & $\mathbf{1 . 2 9 3}$ \\
PIS & 0.147 & 0.140 & 0.113 & 0.143 & 0.127 & 0.119 & 0.122 \\
\hline
\end{tabular}

${ }^{\text {a }}$ Purified recombinant TSPs were used at a concentration of $0.1 \mu \mathrm{g} / \mathrm{ml}$.

${ }^{\mathrm{b}}$ Serum samples were diluted 1:5,000 in 3\% BSA/PBS.

${ }^{\mathrm{c}} \mathrm{PIS}$, Pre-immune sera, mean $\pm \mathrm{SE}=0.130 \pm 0.013$. This is used as a cut-off point (threshold value) to discriminate between "positive" and "negative". Positive values were presented in bold letters.

OD value was measured at A450nm.

TSP, tetraspanin; TRX, thioredoxin. 
Table 4. Number of cyst lesions in mouse liver and percentage reduction in cyst lesions in vaccinated groups compared with non-vaccinated control.

\begin{tabular}{|c|c|c|c|c|c|}
\hline \multirow[t]{2}{*}{ Group } & \multicolumn{3}{|c|}{ Number of cyst lesions } & \multicolumn{2}{|c|}{ Vaccine efficacy $(\%)$} \\
\hline & Mean \pm S.E & Range & $\begin{array}{l}\text { Ratio of mice } \\
\text { without lesions }\end{array}$ & $\begin{array}{l}\text { Cyst lesion } \\
\text { reduction rate }\end{array}$ & Protective rate \\
\hline $\begin{array}{l}\text { Non-vaccinated } \\
\text { control }(n=6)^{d}\end{array}$ & $19.00 \pm 3.1$ & $7-25$ & $0 / 6$ & - & 0 \\
\hline $\operatorname{PBS}(n=7)$ & $20.28 \pm 1.2^{\mathrm{c}}$ & $16-26$ & $0 / 7$ & -6.8 & 0 \\
\hline $\operatorname{TRX}(\mathrm{n}=7)$ & $13.71 \pm 3.5^{\mathrm{c}}$ & $7-34$ & $0 / 7$ & 27.8 & 0 \\
\hline TSP1 $(n=6)$ & $2.33 \pm 1.1^{\mathrm{a}}$ & $0-7$ & $2 / 6$ & 87.9 & 33.3 \\
\hline TSP2 $(n=6)$ & $6.50 \pm 2.7^{b}$ & $0-18$ & $1 / 6$ & 65.8 & 16.7 \\
\hline TSP3 $(n=6)$ & $2.83 \pm 1.5^{\mathrm{a}}$ & $0-8$ & $2 / 6$ & 85.1 & 33.3 \\
\hline TSP4 $(n=7)$ & $6.29 \pm 1.7^{\mathrm{a}}$ & $0-14$ & $1 / 7$ & 66.9 & 14.3 \\
\hline TSP5 (n=7) & $5.00 \pm 0.9^{\mathrm{a}}$ & $2-8$ & $0 / 7$ & 73.7 & 0 \\
\hline TSP6 $(n=6)$ & $5.50 \pm 1.8^{\mathrm{a}}$ & $1-13$ & $0 / 6$ & 72.9 & 0 \\
\hline TSP7 $(n=7)$ & $11.85 \pm 2.9^{\mathrm{c}}$ & $1-27$ & $0 / 7$ & 37.6 & 0 \\
\hline
\end{tabular}

${ }^{\mathrm{a}}$ Reduction of cyst numbers was significant at $\mathrm{P}<0.01$.

${ }^{\mathrm{b}}$ Reduction of cyst numbers was significant at $\mathrm{P}<0.05$.

${ }^{\mathrm{c}}$ Reduction of cyst numbers was not significant $(\mathrm{P}>0.05)$.

${ }^{\mathrm{d}} \mathrm{n}$ represents the number of mice in each group. 


\section{Figure legends}

Fig. 1. Comparison of the LEL domain amino acid sequences for the seven cloned transmembrane proteins (Em-TSP1 to TSP7) and those of Sm-TSP1, Sm-TSP2, Sm23, Sj23 and Ts-T24. Alignment of these amino acid sequences was performed with the Clustal W2 on-line service. Identical amino acid residues are marked with colored shadow. Key residues are marked with a red frame. Extra conserved residues in Schistosoma, Echinococcus and Taenia are marked with a black frame. Echinococcus and Taenia specifically conserved amino acids are in shorter black frames. Em, Echinococcus multilocularis; Sm, Schistosoma mansoni; Sj, Schistosoma japonicum; Ts, Taenia solium; The GenBank (http://www.ncbi.nlm.nih.gov/Genbank) accession numbers for $E$. multilocularis tetraspanin 1 to 7 and Sm-TSP1, Sm-TSP2, Sm23, Sj23 and Ts-T24 are $\underline{\text { FJ384717 }}, \underline{\text { FJ384718 }}, \underline{\text { FJ384719 }}, \underline{\text { FJ384720 }}, \underline{\text { FJ384721 }}, \underline{\text { FJ384722 }}, \underline{\text { FJ384716 }}, \underline{\text { AF521093 }}$ $\underline{\mathrm{AF} 521091}, \underline{\mathrm{M} 34453}, \underline{\mathrm{M} 63706}$ and $\underline{\mathrm{AY} 211879}$ respectively.

Fig. 2. Phylogenetic tree of transmembrane proteins constructed by the MegAlign component of the DNAStar program (Version 4.01 DNASTAR, Madison, Wis.). TSP1 to TSP7: tetraspanins of E. multilocularis (in marked frame); Sm: Schistosoma mansoni; $\mathrm{Sj}$ : 
Schistosoma japonicum; Ts: Taenia solium; Sh: Schistosoma haematobium. GenBank accession numbers for Sm25, Sh23 and Sj25 are AF028730, U23771 and U77941 respectively.

Fig. 3. Amplification of tetraspanins by RT-PCR. (A) LELs of tetraspanins from the cDNA library of E. multilocularis metacestodes with sense and antisense primers designed from the large extracellular loop domain. (B) Investigation of tetraspanins from cDNA of oncosphere cDNA with forward and antisense primers designed from ORFs of full-length tetraspanins. In both (A) and (B), Lane M, molecular marker. Lanes 1 to 7, TSP1 to TSP7.

Fig. 4. SDS-PAGE analysis of purified recombinant TSPs. Purified recombinant TSPs were loaded at 5-10 $\mu \mathrm{g} /$ well and separated on a $12 \%$ SDS-polyacrylamide gel. CBB staining was performed. Lane M, molecular marker. Lanes 1 to 7, TSP1 to TSP7. Lane 8, purified thioredoxin (TRX).

Fig. 5. Immunoblotting analysis of anti-TSP sera (TRX-removed) to cyst-extracted antigens. Serum samples were diluted 500-fold and the anti-rabbit IgG-HRP conjugate was diluted 5,000-fold with blocking buffer. Pre-immune serum was used as the negative control. Lane M, molecular marker. Lanes 1 to 7 , reactivity of cyst-extracted antigens to anti-TSP1 to 
TSP7 antibodies. Lane 8, negative control.

Fig. 6. Reactivity of recombinant TSP-immunized-mice sera (TRX-absorbed) to recombinant TSPs and cyst-extracted antigens measured by ELISA. Serum samples were diluted 5,000-fold and the goat anti-mouse-IgG-HRP conjugate was diluted 10,000-fold in blocking buffer. For each group, a, b represents reaction with recombinant TSP (symbol•) and cyst-extracted antigen respectively (symbol $)$ ). The mean OD value of control reaction where TRX had been used as antigen (range of the mean values among TSP1-TSP7: 0.05-0.12) was subtracted from each group. Vertical bars represent mean OD value of each group. $\mathrm{N}$, number of mice in each group. 


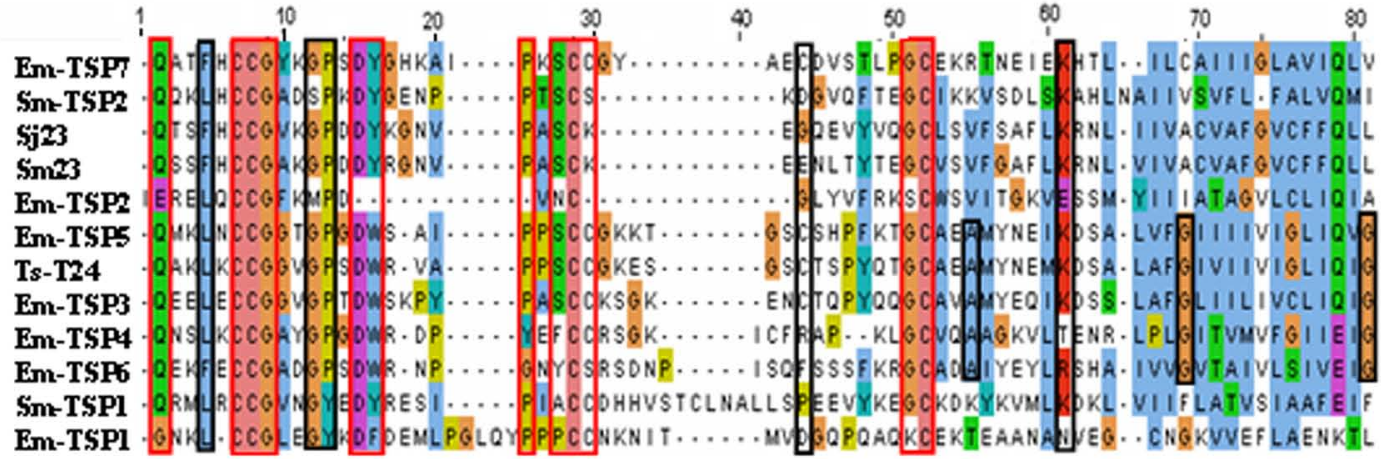




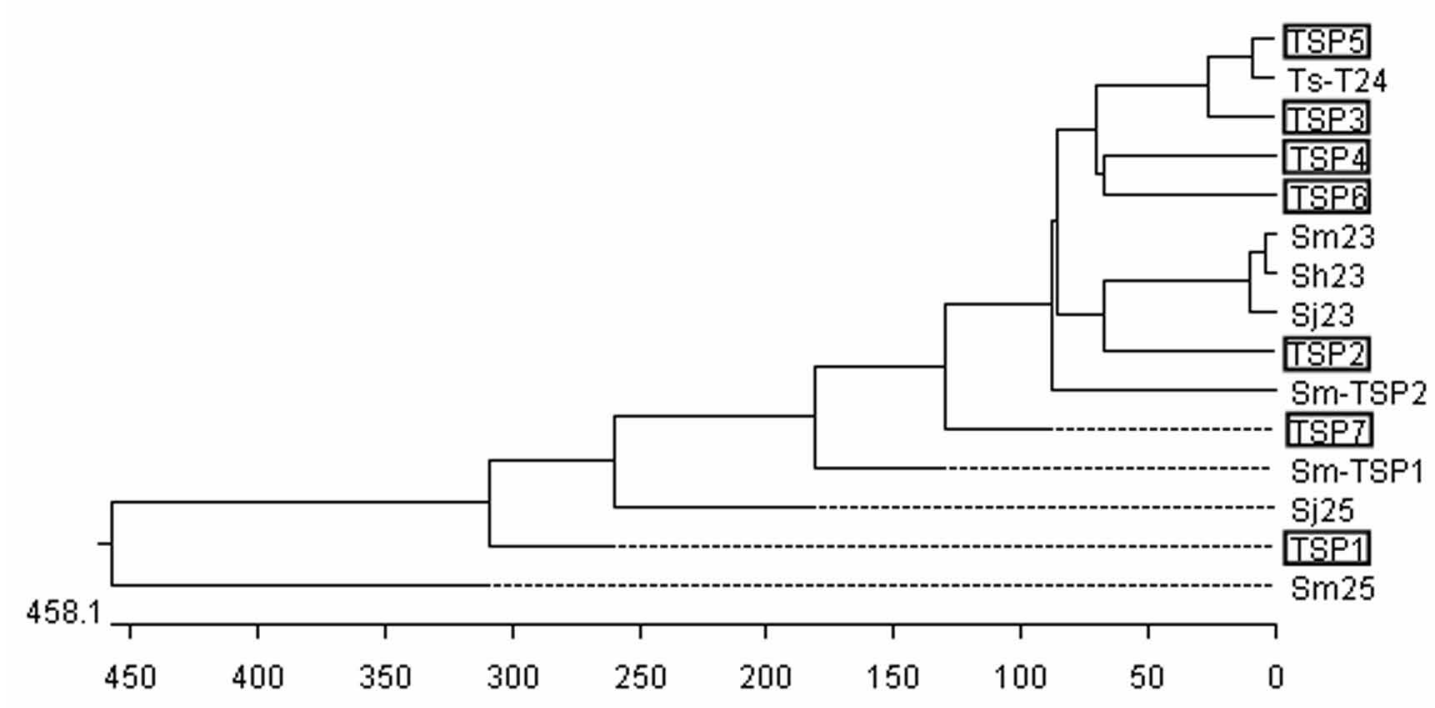


(A)

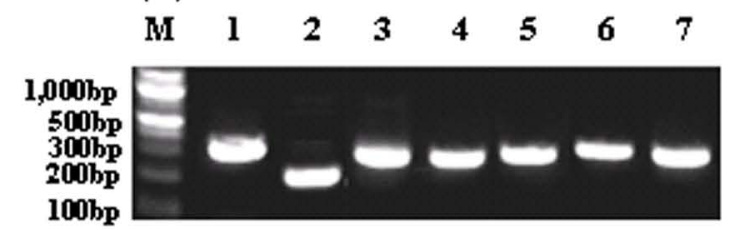

(B)

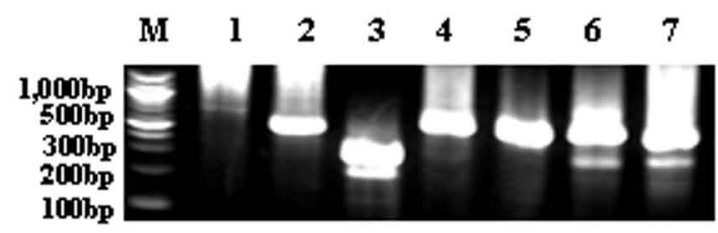




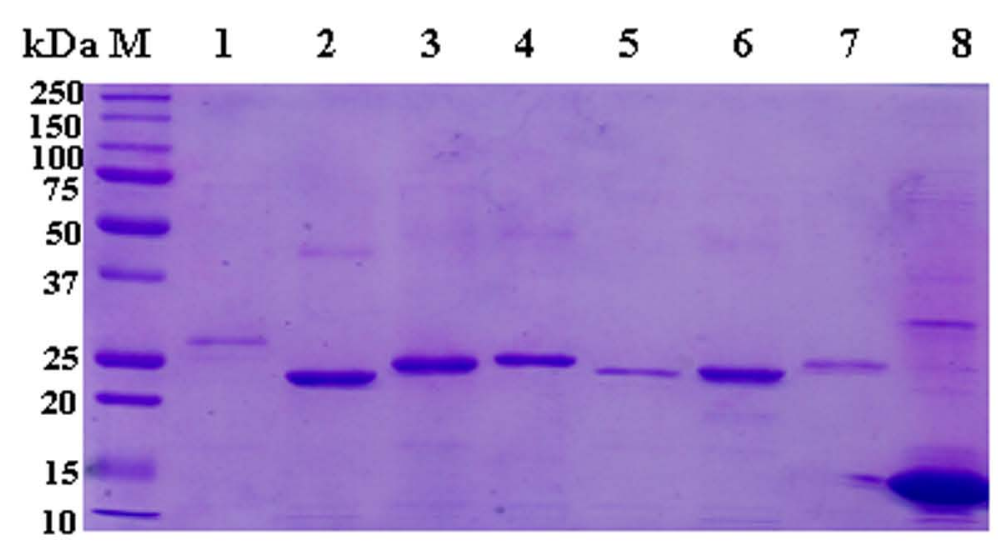




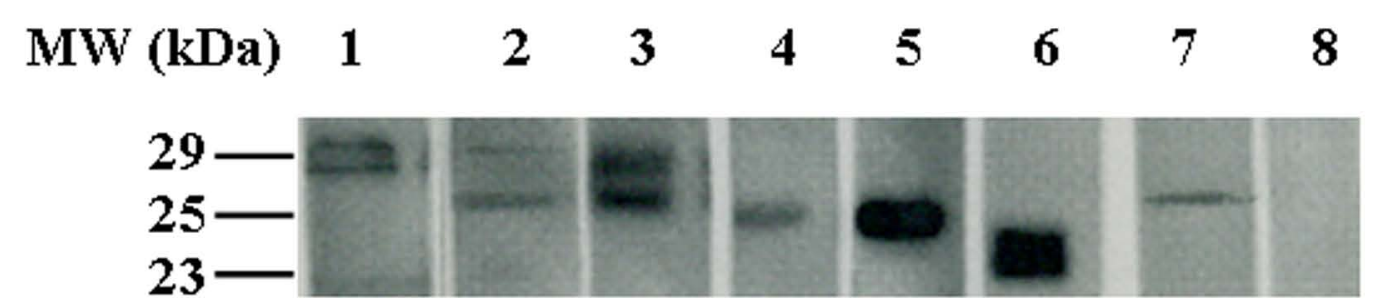




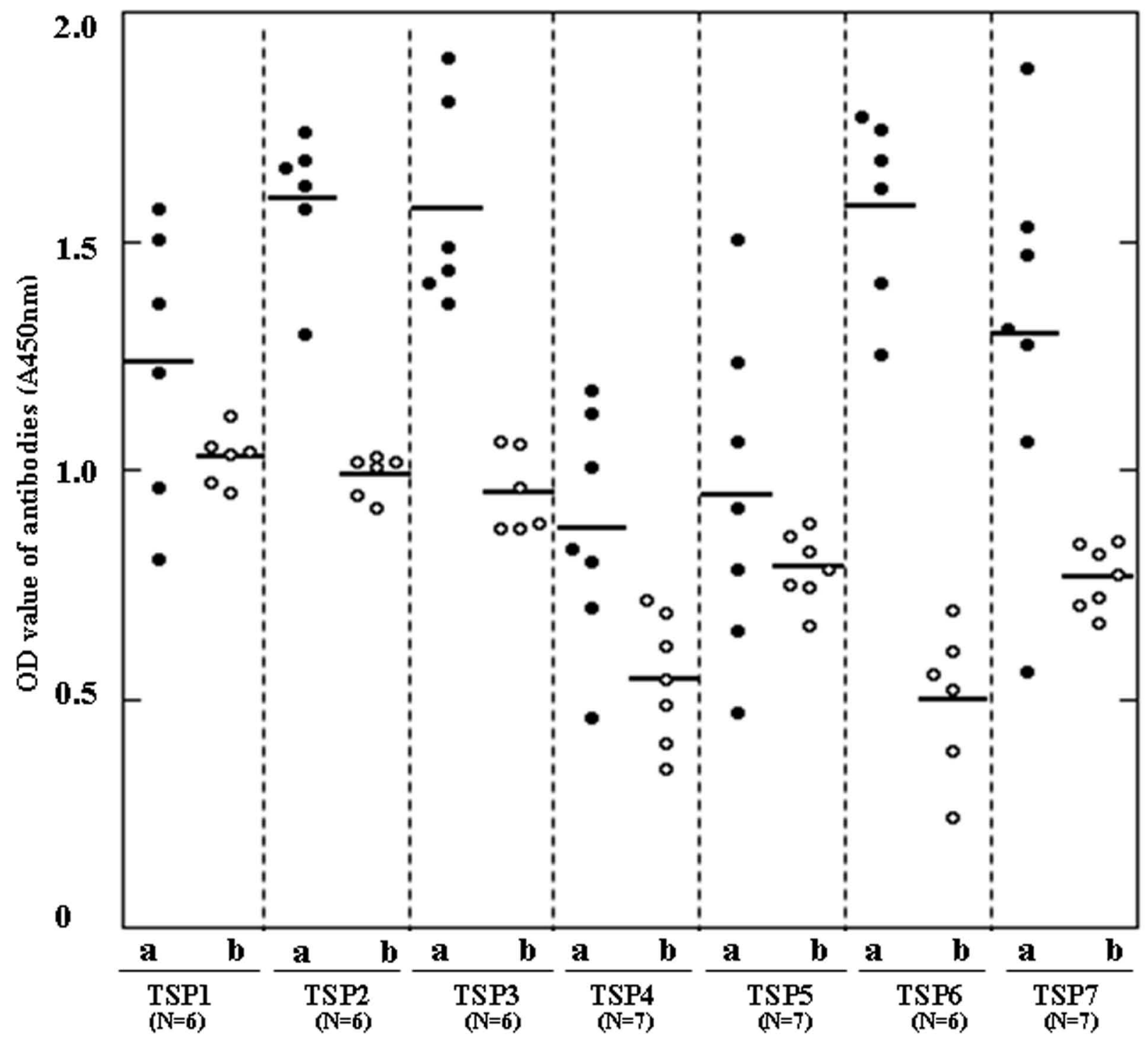

